\title{
BMJ Open Understanding the lived experiences of severe postnatal psychiatric illnesses in English speaking South Asian women, living in the UK: a qualitative study protocol
}

\author{
Harpreet Kaur Sihre, ${ }^{\oplus 1}$ Paramjit Gill, ${ }^{2}$ Antje Lindenmeyer, ${ }^{3}$ Mary McGuiness, ${ }^{4}$ \\ Giles Berrisford, ${ }^{4}$ Jelena Jankovic, ${ }^{4}$ Minaxi Patel, ${ }^{5}$ Jona Lewin, ${ }^{5}$ Qulsom Fazil ${ }^{3}$
}

To cite: Sihre HK, Gill P, Lindenmeyer $A$, et al. Understanding the lived experiences of severe postnatal psychiatric illnesses in English speaking South Asian women, living in the UK: a qualitative study protocol. BMJ Open 2019;9:e025928. doi:10.1136/ bmjopen-2018-025928

\section{- Prepublication history for} this paper is available online. To view these files, please visit the journal online (http://dx.doi org/10.1136/bmjopen-2018025928).

Received 08 August 2018

Revised 03 July 2019

Accepted 05 July 2019
Check for updates

(C) Author(s) (or their employer(s)) 2019. Re-use permitted under CC BY-NC. No commercial re-use. See rights and permissions. Published by BMJ.

For numbered affiliations see end of article.

Correspondence to Harpreet Kaur Sihre; hks449@student.bham.ac.uk

\section{ABSTRACT}

Introduction The South Asian population is the UK's largest and fastest growing minority ethnic group. There is evidence to suggest the lay understanding of postnatal psychiatric illnesses of this group may fall outside the purview of Western biomedical perspectives. Alternative explanations include psychosocial, cultural and spiritual factors. Approaching psychiatric illnesses through a social perspective includes gaining insight to the patient's subjective experiences and understandings via qualitative inquiry. The objectives of this qualitative study are to explore South Asian women's narrative of living with a severe postnatal psychiatric illness and experiences of Perinatal Mental Health Services, care and support.

Methods and analysis Data collection is ongoing and will continue until 31 December 2018. Participants will be identified and recruited from Perinatal Mental Health Services in Birmingham and London. Eligible participants will be English speaking South Asian females aged 18 years or above with the capacity to give written informed consent. Participants are clinically diagnosed with a severe postnatal psychiatric illness. This qualitative study uses individual in-depth face-to-face interviews that aim to last 1 hour. Interviews will be audio recorded with participants' permission. Interview audio recordings will be transcribed verbatim and analysed using interpretative phenomenological analysis (IPA). The primary goal of IPA is for the researcher to closely study and interpret how individuals make sense of their life experiences in a particular context by drawing on the fundamental principles of phenomenology, hermeneutics and idiography.

Ethics and dissemination The University of Birmingham, the South Birmingham Research Ethics Committee and the Health Research Authority have approved this study (approvals date: 18-12-2017 ref: 17/WM/0350). Local capability and capacity have been confirmed from Trust Research and Development departments. The researchers plan to publish the results from this study in journals and present findings at academic conferences.
Strengths and limitations of this study

- First interpretative phenomenological analysis (IPA) study to investigate South Asian women's lived experiences of severe postnatal psychiatric illness, living in the UK.

- The purpose of using IPA is to understand and interpret the subjective meaning of a social phenomenon. Lived experiences takes into account the social and cultural milieu of the individual lives.

- Results will inform the development of culturally competent care.

- Limitations: a small selective sample means findings will not be generlisable.

- Excluding non-English speaking South Asian women will not highlight the different experiences are likely to have in comparison of English speaking South Asian women.

\section{SEVERE POSTNATAL PSYCHIATRIC ILLNESSES}

Postnatal psychiatric illnesses occur within the year after childbirth and are one of the leading cause of maternal morbidity in the UK. ${ }^{1-3}$ Postnatal depression (PND) is a non-psychotic depressive disorder that occurs in $\sim 10 \%$ of new mothers in high-income countries, with increased estimates in lower-middle-income countries. ${ }^{4}$ Postnatal psychosis the most severe type of illness, documented to impact 2 in every 1000 deliveries. ${ }^{6}$ A further 2 per 1000 new mothers will suffer from another form of severe psychological state including severe depression, schizophrenia and bipolar disorder. ${ }^{1}$ Although the incidence is small, the impact can be devastating for the mother, the infant, the partner and the family. ${ }^{78}$ It is documented that women with a history of bipolar disorder or a postnatal psychotic episode are at an elevated risk for postnatal relapse. ${ }^{8} 9$ Furthermore, 
changes in medication in women with pre-existing severe psychiatric illnesses may be at a higher risk for postnatal relapse. ${ }^{10}$ Symptom onset of severe illnesses can occur rapidly and unexpectedly during the early days or weeks following childbirth.

\section{Postnatal psychosis}

The clinical features of postnatal psychosis can be dramatic and include mania, psychotic thoughts and severe depressive symptoms. ${ }^{11}{ }^{12}$ Women may feel excited or elated, the inability or not needing to sleep. ${ }^{12}{ }^{13}$ Women may also develop paranoia and bewilderment. ${ }^{1}$ Frequent obsession thoughts of infanticide may be present, which often centre on the baby resulting in impulses to harm the baby or oneself as a command of auditory hallucinations. ${ }^{14}$

\section{Severe depression}

Severe depression is presented by feelings of guilt, psychomotor slowing and agitation, high level of cognitive disorders, anxiousness and feelings of hopelessness, hyprochronidria and somatic symptoms. ${ }^{15} \mathrm{~A}$ small number of women with severe depression are likely to develop psychotic symptoms including hearing voices and have unusual beliefs. Neonaticide is rare in mothers with severe depression; however, women with thoughts of harming their infants are more likely to act on them. ${ }^{16} 17$

\section{Schizophrenia}

Symptoms of postnatal schizophrenia can be debilitating and include social withdrawal, cognitive disorganisation, misinterpretation of reality, hallucinations, seeing things that others cannot seeing. Cognitive symptoms include, difficulty in focusing attention, memory problems and difficulty maintaining conversation. ${ }^{18}$ It should be noteworthy that schizophrenia may arise prior to conception or during pregnancy and symptoms may continue into the postnatal period. ${ }^{19}$

\section{Postnatal bipolar disorder}

Bipolar disorder is typically characterised by a wide range of mood changes carrying between manic episodes, hypomania or episodes of depression. ${ }^{20}$ Postpartum psychosis may be the first presentation of bipolar disorder. ${ }^{19}$

\section{Specialist Perinatal Mental Health Services}

Women experiencing severe postnatal psychiatric illnesses typically require hospitalisation and treatment via Specialist Perinatal Mental Health Services. ${ }^{121}$ Specialist Perinatal Mental Health Services are for women experiencing a severe and enduring types of psychiatric illness after childbirth. These include Specialist Community Perinatal Mental Health Teams and Mother and Baby Units (MBU). Secondary care consists of Specialist Community Perinatal Mental Health Teams on referral by a primary care physician, who offer specialist biopsychosocial assessments and care plans. A MBU is a specialist inpatient psychiatric tertiary care service with several beds for joint admission of a very severely ill mother and her baby. The Independent Mental Health Taskforce to the
NHS in England (2016) reported the Five-Year Forward View Plan for Mental Health. ${ }^{22}$ Support for new mothers and babies during the postnatal period was reported as a specific theme. As set out in the report, there has been unprecedented investment into Specialist Perinatal Mental Health Services. £365 million is being invested in Specialist Perinatal Mental Health Services, so that all women across the region can have access to evidencebased treatment. ${ }^{23}$

Historically, psychiatry was considered a biomedical concept, in isolation of social and cultural determinants. ${ }^{24}$ However, recent findings suggest the understandings and causes of postnatal psychiatric illness are multifactorial and are viewed through a psychosocial, sociocultural and religious/spiritual lens. ${ }^{25}$ Although there are many existing theoretical concepts that aim to understand postnatal psychiatric illness, recently, light has been given to the patient perspective in order to understand and conceptualise their illness experiences. However, the literature surrounding women's understandings and experiences of their psychiatric illness after childbirth remains underdeveloped. Exploring the social origins of illness via the subjective belief system of the patient and their experiences of illness is also clinically relevant for assessment, diagnosis and management. ${ }^{26} 27 \mathrm{~A}$ small body of qualitative studies have explored the cause and consequence, women's experiences of their illness, women's experiences of treatment and support and recovery needs, experience in relation to the stigmatisation of their postnatal psychiatric illness. ${ }^{781228-30}$ However, the majority of these studies were conducted in Western Europe, thus limiting cross-cultural generlisability of findings. Studies that use samples from the 'general population' often exclude non-Western population groups and do not capture the variety of conceptualisations of illness from different cultural groups.

Super diversity has become a 21st century phenomena in the UK. A super diverse nation is characterised by individuals who originate from a range of countries and ethnicities, which include heterogeneous cultures, religions and languages. ${ }^{31}$ The South Asian population are the largest and fastest growing minority ethnic group. ${ }^{32}$ The 2011 UK Census identifies Indian, Pakistani and Bangladeshi ethnicities within the South Asian ethnic group and for the sake of brevity, 'South Asian' will be used throughout this protocol. ${ }^{33}$ Individuals with South Asian origins often share similarities in relation to their cultural beliefs, norms and values. ${ }^{34}$ Although the prevalence rates of severe postnatal psychiatric illnesses in the South Asian population is under-researched, there are quantitative data to suggest that South Asian women are more susceptible to developing postnatal psychiatric illness than their White counterparts. ${ }^{35}{ }^{36}$ Previous literature on South Asian populations has predominantly focused on PND and identified determinants including husband/marital relationship problems, conflict with in-laws, dissatisfaction with infant's gender, financial difficulties, lack of social support, culturally specific 
postpartum rituals and language and communication barrier. ${ }^{37} 38$ Although some factors associated with PND overlap Western conceptualisations, some differences in ethnicity, culture and society pervade through South Asian women and recognised as significant risk factors for developing PND. Only one study was identified in India that investigates severe postpartum psychiatric illnesses in South Asian women. ${ }^{39}$ Almost the majority of women in this study reported infanticide ideas and behaviours, separation from the infant and psychotic ideas towards the infant. ${ }^{39}$ Thoughts of infanticide were associated with the birth of a female, a concept that was not present among Western population groups. ${ }^{39}$

Although there is considerable heterogeneity within and between the South Asian populations, cultural similarities contribute to common challenges when South Asian populations are seen in primary and secondary healthcare settings. We know that cultural and religious beliefs often contribute towards the understanding of mental illness and so investigating associations in specific ethnic groups is important in promoting equalities in mental healthcare provision. This information is crucial to understand how culture, religious and social context contributes to severe mental illness. For example, Hussain and Cochrane investigated beliefs on causes and cures in clinically depressed South Asian women living in Birmingham. ${ }^{40}$ This study concluded that cultural and religious beliefs exert an influence in South Asian women's beliefs about the understanding, causes and cures of their mental illness and were central to these women's experiences of depression. ${ }^{40}$ Culture and religion beliefs can be fluid and can structure the way a person defines illness and how or where treatment is sought that may influence the management of illness. To our knowledge, no study has explored experiences of severe postnatal psychiatric illnesses in South Asian women residing in the UK. Therefore, the overall goal of this research study is to provide a deeper understanding of the lived experiences of South Asian women diagnosed with a severe postnatal psychiatric illness.

\section{Research objectives}

1. To explore South Asian women's experiences of living with a severe postnatal psychiatric illness.

2. To explore South Asian women's experiences of Perinatal Mental Health Services, care and support.

\section{METHODS AND ANALYSIS \\ Study design}

Qualitative methods using interpretative phenomenological analysis (IPA) is appropriate for this study. IPA is a methodological framework in qualitative research that focuses on exploring in-depth how individuals make sense of their lived experiences, including how they experience living through events and what meaning they attribute to a given phenomenon. ${ }^{41}$ IPA is invaluable when discussing emotional and sensitive topics, such as psychiatric illnesses. IPA enables the participant to retrospectively provide a full detailed account of their experiences with the researcher remaining strongly empathetic and highly attuned to their personal accounts. As we are interested in lived experiences, IPA allows a micro reading of the participant's accounts, leading to deeper understandings of the phenomena. With theoretical origins in phenomenology, hermeneutics and ideography, IPA allows the researcher to rigorously explore and interpret how a specific experience may affect a person in the context of their lives in a under-developed research area. ${ }^{41}$

\section{Patient and public involvement}

The researcher approached patients on the MBU and asked if they were interested to provide their views and opinions about the research study through an informal consultation. A South Asian patient agreed to give their views and opinions. The patient was presented with a brief overview of the research protocol. The patient was asked to provide their views about the appropriateness of the research methods in obtaining information about experiences of postnatal psychiatric illness.

The patient was also shown the research materials and asked to provide their views, feedback and any other comments on how they could be improved. Research materials included letter of invitation to participant, participant information sheet, participant consent form, interview schedule and letter to GP. Changes to the research materials were implemented based on the patient's comments. For example, the patient commented that some terms used as part of the interview schedule might not be understood. These terms were rephrased with the patient in a way that would be understandable.

\section{Sampling strategy}

A non-probabilistic, purposive sampling strategy is appropriate for IPA studies. The participants will be purposely selected as they have 'lived experience' about the phenomena being explored. Therefore, due to the deliberate choice of participants, the data drawn will contribute to a better understanding about a particular group and the phenomena under investigation. ${ }^{42}$ The demographic population of interest purposively recruited for this study are women from a South Asian population (Bangladeshi, Indian and Pakistani), any generation. This group also includes major religious groups including Muslims, Hindus and Sikhs.

\section{Eligibility criteria}

English speaking South Asian new mothers who have been diagnosed with a severe postnatal psychiatric and currently presenting or presented within a year at Perinatal Mental Health Services will be considered for this study. The participant may be eligible for the study if the following criteria apply:

1. Willing and capacity to give informed consent for participation in the study. Prior to informed consent, capacity for participants to potentially partake in the 
study will be determined by a member of their clinical care team.

2. South Asian female (Indian/Bangladeshi/Pakistani)

3. Clinically diagnosed with a severe postnatal psychiatric illness by a perinatal psychiatrist and is/has received care from Perinatal Mental Health Services (this includes specialist community perinatal mental health teams and MBUs).

4. Women must be at 3 months' discharge before interview takes place (only applies for in-patients).

5. Their illness is an extension from pregnancy or as a new episode within the year after childbirth.

6. 18 years of age or above.

7. Able to speak sufficient English to take part. We will not be including women non-English speaking women due to lack of resources.

\section{Participant identification and recruitment}

All participants will be identified and recruited from two Perinatal Mental Health Services in Birmingham and London. The multidisciplinary clinical care team (MDT) will be responsible for identifying all potential participants and initial approach. The MDT team comprise of perinatal psychiatrists, clinical psychologists, clinical nurse specialists, nursing staff, occupational therapists, perinatal psychiatric nurses and administrative staff. If potential participants preliminary express an interest to partake and agree for their details to be given to the researcher, the researcher will continue with recruitment. Participants will have the option for the interview to take place at their home, the NHS Trust, the university or a preferred quiet location. A participant information sheet will be administered to the participant prior to the interview. An opportunity to ask questions will be given to the potential participant.

Potential participants are being contacted through the following routes:

1. A telephone call.

2. Inpatients identified on the MBU who fit eligibility will be approached at discharge.

3. A letter of invitation to participants discharged up to a year.

\section{Sample size}

There is no necessary correct number of participants for an IPA qualitative study. ${ }^{41}$ The primary concern of IPA research is to elicit rich and detailed accounts of a specific inquiry and maintain and manage the in-depth analysis of key individuals. ${ }^{41}$ IPA study sample sizes are typically small so that the amount of data collected will not be overwhelming. We will not aim to reach data saturation sampling. We will stop recruitment of participants and data collection when we trust we have sufficient data and participants to conduct an IPA analysis. Due to the small patient cohort, we envisage to recruit between five and eight participants for this research study.

\section{Data collection}

Demographic characteristics will be collected prior to the interview. Data will be collected using qualitative semistructured, face-to-face interviews by the first author. HKS has received formal training in qualitative research methods. An interview schedule has been developed from a literature review and in relation to the research questions, which includes understanding of the illness, lived experiences, illness beliefs, perceived causes and experiences of treatment and care. The interview schedule includes the following topics:

1. Diagnostic history.

2. Understanding of the illness.

3. Illness beliefs and perceived causes.

4. Lived experiences of a severe postnatal psychiatric illness.

5. The journeys, pathways and process of referral and admission.

6. Experiences of Perinatal Mental Health Services and support networks

The interview schedule includes a range of open-ended questions to encourage the interviewee to talk in detail about their experiences, which allows the interviewee to set the parameter. The duration of the interview aims to last $\sim 1$ hour. With participants' permission, interviews will be recorded on a digital voice recorder and supplemented by additional field notes. Interviewees will be provided with choice of where to be interviewed including their homes, the trust, the university or a convenient location.

\section{DATA ANALYSIS}

Each interview will be transcribed verbatim before the analysis. The interview transcripts will serve as the raw data to be analysed following the principles of IPA. ${ }^{41}$ The principles of IPA include phenomenology, hermeneutics and idiography. ${ }^{43}$ Phenomenology, influenced by Husserl ${ }^{44}$, is the study of consciousness through in-depth descriptions of a lived experience. Hermeneutics is the theory of interpretation of the lived experience, developed primarily by Heidegger. ${ }^{45}$ Idiography then focuses on the particular experience of the individual and their specific context. Participant names will be replaced by an ID number to ensure their anonymity. Initially, IPA involves close readings of transcripts where initial thoughts, observations and responses to the text will be noted in relation to the research objective. The researcher will try to step into the participant's shoes as deeply as possible. To ensure reflexivity as a method of validity, as part of the process of IPA, the first author will deliberately put to aside (bracket) their own experiences, values, preconceptions and beliefs during the initial stages of analysis, which may influence the interpretation of the participant's responses. ${ }^{46}$ Distinctive phrases and emotional responses will also be highlighted at this initial stage of analysis. Second, the researcher will work more closely with the detailed notes that have been developed from the initial readings. Using these comprehensive notes, the researcher will aim 
to formulate concise phrases through a psychological lends that capture an interpretation and understanding of the participant's experiences, thoughts and emotions. The next stage involves seeking connexions between emerging themes and grouping them together according to conceptual and theoretical similarities. Each theme or cluster will be given a descriptive label. This process will be repeated for each transcript and patterns will be established between individual cases on a master table. These may include convergences or divergences between themes that developed from each transcript. The themes identified in the final master table will be transformed into a narrative account exemplified by verbatim quotes from the original scripts. The analysis will be managed with software assistance Nvivo.

\section{Ethics and dissemination}

Benefits to participants

Living with a severe mental illness is often said to decrease self-esteem and confidence, as it is still viewed as a taboo subject in the South Asian culture. By reflecting on their mental illness, participants will be able to fully explore their illness any may also gain a new perspective. This sense of self-awareness and self-exploration may be empowering for the participant. The data collected may be shared with others through presentations, publications and conferences. To be able to understand and increase an awareness of their illness may also help other South Asian women to accept and understand their illness.

\section{Participant withdrawal}

Participants will have the right to withdraw before or during the interview; however, a cut-off point of 2 weeks after the interview will be added to the withdrawal of data.

\section{Deception}

Participants will not be deceived in any way about study objectives. All information regarding the study will be provided verbally and in writing prior to the interview.

\section{Consent}

Eligible participants must have the full capacity to provide informed consent for themselves. Verbal and written informed consent is obtained from all those who are directly involved in the research, after they have been informed about the following issues: the purpose of the study, the types of data and methods of data collection, confidentiality and anonymity conditions associated with the data, time commitment expected from participants, the right to decline information, the opportunity to withdraw at any time during the interview, details of any risks, any plans for debriefing, how the data will be used, benefits of the research and how the results of research can be made available to participants. At least 24 hours is given to potential participants to absorb and to consider the information provided on the participant information sheet. An opportunity to ask any relevant questions is given before they provide consent.

\section{Confidentiality}

Data obtained from the participant will be kept confidential. Participants will remain anonymous and their names will be replaced by an ID number during transcription and analysis. When disseminating findings from the research study, direct quotes from respondents may be used as an example and will remain anonymous.

\section{Psychological discomfort and distress}

We envisage that recalling a distressful or traumatic event may cause some discomfort and suffering for the participant. If at any point the participant feels discomforted, distressed or upset during the interview, they will be given the option to pause the interview and continue at a time they feel more comfortable. If they feel they no longer wish to participate, they will have the opportunity and right to withdraw. The researcher will have a file of support services that they can provide to the participant during the interview. Furthermore, the researcher will use their own judgement to terminate the interview if the participant seems too ill to partake on initial meeting. If during the interview the participant mentions current thoughts of harm or suicide to self and/or others, relapse, malpractice or abuse, this information will be disclosed to the clinical team for the safety of the participant. This information will be disclosed to protect the health and safety of the mother, baby and any household members. Disclosure of the above mentioned information will be discussed during the consent process and is clearly mentioned on the participation information sheet,

\section{Storage of personal data}

Manual files such as informed consent sheets will contain personal information. These manual files will be kept in a secure filing cabinet at the university that has been allocated to the researcher for the duration of the study, who will only have access. Any data collected from the interview that was recorded on the dictaphone will be transferred onto the researcher's secure university account. Data will be stored anonymously and archived on the University's Research Data Archive for 10 years.

\section{DISSEMINATION}

The researchers will prepare manuscripts and publish the results from this study in relevant peer-reviewed journals and present findings via poster presentations and oral presentations at appropriate academic and non-scientific conferences.

\section{Author affiliations \\ ${ }^{1}$ Institute of Applied Health Research, University of Birmingham, Birmingham, UK ${ }^{2}$ Social Science and Systems in Health, University of Warwick, Warwick, UK ${ }^{3}$ Institute of Applied Health Research, University of Birmingham, Birmingham, UK ${ }^{4}$ Perinatal Mental Health services, Birmingham and Solihull Mental Health Foundation Trust, Birmingham, UK \\ ${ }^{5}$ Coombe Wood Perinatal Service, Central and North West London Foundation Trust, London, UK}

Acknowledgements We sincerely thank the clinical team at Birmingham and Solihull Mental Health Foundation Trust Perinatal Mental Health Services 
and Central and North West London Perinatal Mental Health Services for their collaboration and support for the study. We would also like to sincerely thank our patient advisor who provided insightful and invaluable comments to research materials.

Contributors HKS, QF, PG, AL, MM and GB conceptualised the study. HKS wrote the manuscript. QF, AL, PG, MM, GB, JJ, MP and JL participated in revising and approving the final manuscript.

Funding The authors have not declared a specific grant for this research from any funding agency in the public, commercial or not-for-profit sectors.

Competing interests None declared.

Patient consent for publication Not required.

Provenance and peer review Not commissioned; externally peer reviewed.

Open access This is an open access article distributed in accordance with the Creative Commons Attribution Non Commercial (CC BY-NC 4.0) license, which permits others to distribute, remix, adapt, build upon this work non-commercially, and license their derivative works on different terms, provided the original work is properly cited, appropriate credit is given, any changes made indicated, and the use is non-commercial. See: http://creativecommons.org/licenses/by-nc/4.0/.

\section{REFERENCES}

1. Paschetta E, Berrisford G, Coccia F, et al. Perinatal psychiatric disorders: an overview. Am J Obstet Gynecol 2014;210:501-9.

2. Oates M. Perinatal psychiatric disorders: a leading cause of materna morbidity and mortality. Br Med Bull 2003;67:219-29.

3. Knight M. The findings of the MBRRACE-UK confidential enquiry into maternal deaths and morbidity. Obstetrics, Gynaecology \& Reproductive Medicine 2019;29:21-3.

4. Howard LM, Molyneaux E, Dennis C-L, et al. Non-psychotic menta disorders in the perinatal period. The Lancet 2014;384:1775-88.

5. Fisher J, Cabral de Mello M, Patel V, et al. Prevalence and determinants of common perinatal mental disorders in women in low- and lower-middle-income countries: a systematic review. Bull World Health Organ 2012;90:139-49.

6. Gale S, Harlow BL. Postpartum mood disorders: a review of clinical and epidemiological factors. J Psychosom Obstet Gynaecol 2003;24:257-66.

7. Doucet S, Letourneau N, Blackmore ER. Support needs of mothers who experience postpartum psychosis and their partners. J Obstet Gynecol Neonatal Nurs 2012;41:236-45.

8. Glover L, Jomeen J, Urquhart T, et al. Puerperal psychosis - a qualitative study of women's experiences. J Reprod Infant Psychol 2014;32:254-69.

9. Wesseloo R, Kamperman AM, Munk-Olsen T, et al. Risk of postpartum relapse in bipolar disorder and postpartum psychosis: a systematic review and meta-analysis. American Journal of Psychiatry 2016;173:117-27.

10. Taylor CL, Stewart RJ, Howard LM. Relapse in the first three months postpartum in women with history of serious mental illness. Schizophr Res 2019;204:46-54.

11. Kendell RE, Chalmers JC, Platz C. Epidemiology of puerperal psychoses. Br J Psychiatry 1987;150:662-73.

12. Heron J, McGuinness M, Blackmore ER, et al. Early postpartum symptoms in puerperal psychosis. BJOG: Int J O \& G 2008;115:348-53.

13. Essali A, Alabed S, Guul A, et al. Preventive interventions for postnatal psychosis. Cochrane Database of Systematic Reviews 2013;7.

14. Sit $D$, Rothschild AJ, Wisner KL. A review of postpartum psychosis. Journal of Women's Health 2006;15:352-68.

15. Zdanowicz A, Wierzbinski P, depression P. Psychiatria i psychologia kliniczna-journal of psychiatry and clinical psychology 2017;17:115-9.

16. Donahue Jennings K, Ross S, Popper S, et al. Thoughts of harming infants in depressed and nondepressed mothers. J Affect Disord 1999;54:21-8.

17. Miller LJ. Postpartum mood disorders. American Psychiatric Association, 1999.

18. Seeman MV. Schizophrenia during pregnancy and the postpartum period. Current Clinical Practice: Psychiatric Disorders in Pregnancy and the Postpartum: Principles and Treatment 2006:139-52.
19. Jones I, Chandra PS, Dazzan P, et al. Bipolar disorder, affective psychosis, and schizophrenia in pregnancy and the post-partum period. The Lancet 2014;384:1789-99.

20. Rusner M, Berg M, Begley C. Bipolar disorder in pregnancy and childbirth: a systematic review of outcomes. BMC Pregnancy Childbirth 2016;16:331.

21. Kissane D, Ball JRB. Postnatal depression and psychosis - a mother and baby unit in a general Hospital. Aust N Z J Obstet Gynaecol 1988;28:208-12.

22. England N. Five year forward view. 20142016.

23. NHS England NI. National collaborating centre for mental health, National collaborating centre for mental. The. England: Perinatal Mental Health Care Pathways. NHS, 2018.

24. Engel $\mathrm{G}$. The need for a new medical model: a challenge for biomedicine. Science 1977;196:129-36.

25. Tovino SA. Scientific understandings of postpartum illness: improving health law and policy. Harv JL \& Gender 2010;33.

26. Allen J, Balfour R, Bell R, et al. Social determinants of mental health. International Review of Psychiatry 2014;26:392-407.

27. Carod-Artal FJ. Social determinants of mental health. Global Mental Health: Springer, 2017: 33-46.

28. Robertson E, Lyons A. Living with puerperal psychosis: a qualitative analysis. Psychol Psychother 2003;76:411-31.

29. Engqvist I, Ferszt G, Åhlin A, et al. Women's experience of postpartum psychotic episodes - analyses of narratives from the Internet. Arch Psychiatr Nurs 2011;25:376-87.

30. Heron J, Gilbert N, Dolman C, et al. Information and support needs during recovery from postpartum psychosis. Arch Womens Ment Health 2012;15:155-65.

31. Phillimore J. Delivering maternity services in an era of superdiversity: the challenges of novelty and newness. Ethn Racial Stud 2015;38:568-82

32. Vertovec S. Super-diversity and its implications. Ethn Racial Stud 2007;30:1024-54.

33. Bradford-ONS S. Ethnicity and national identity in England and Wales 2011, 2012

34. Ahmad F, Shik A, Vanza R, et al. Voices of South Asian women: immigration and mental health. Women Health 2005;40:113-30.

35. Onozawa K, Kumar RC, Adams D, et al. High EPDS scores in women from ethnic minorities living in London. Arch Womens Ment Health 2003;6:s51-5.

36. Edge D. Perinatal mental health care for black and minority ethnic (BME) women: a scoping review of provision in England. Ethnicity and Inequalities in Health and Social Care 2010;3:24-32.

37. Nilaweera I, Doran F, Fisher J, Prevalence FJ. Prevalence, nature and determinants of postpartum mental health problems among women who have migrated from South Asian to high-income countries: a systematic review of the evidence. J Affect Disord 2014;166:213-26

38. Wittkowski A, Zumla A, Glendenning $S$, et al. The experience of postnatal depression in South Asian mothers living in Great Britain: a qualitative study. J Reprod Infant Psychol 2011;29:480-92.

39. Chandra PS, Venkatasubramanian G, Thomas T. Infanticidal ideas and infanticidal behavior in Indian women with severe postpartum psychiatric disorders. J Nerv Ment Dis 2002;190:457-61.

40. Hussain FA, Cochrane R. Depression in South Asian women: Asian women's beliefs on causes and cures. Ment Health Relig Cult 2002;5:285-311.

41. Pietkiewicz I, Smith JA. A practical guide to using interpretative phenomenological analysis in qualitative research psychology. Psychological Journal 2014;20:7-14.

42. Etikan I, Musa SA, Alkassim RS. Comparison of convenience sampling and purposive sampling. American Journal of Theoretical and Applied Statistics 2016;5:1-4

43. J Charlick S, Pincombe J, McKellar L, et al. Making sense of participant experiences: interpretative phenomenological analysis in midwifery research. International Journal of Doctoral Studies 2016;11:205-16.

44. Husserl E. Phenomenology. Encyclopaedia Britannica 1927;14:699-702.

45. Heidegger M. Being and time. 1927. Trans. John Macquarrie and Edward Robinson. New York: Harper, 1962.

46. Chan ZC, Y-I F, W-t C. Bracketing in phenomenology: only undertaken in the data collection and analysis process. The qualitative report 2013;18:1-9. 